Dobrzyński Michal, Czarnota Jakub, Skubel Tomasz, Sokół Dorota, Dudek Iga, Drozd Małgorzata, Piecewicz-Szczęsna Halina. Intravascular lithotripsy in the treatment of peripheral artery disease. Journal of Education, Health and Sport. 2020;10(9):278-283. eISSN 2391-8306. DOI http://dx.doi.org/10.12775/JEHS.2020.10.09.031

https://apcz.umk.pl/czasopisma/index.php/JEHS/article/view/JEHS.2020.10.09.031

https://zenodo.org/record/4026058

The journal has had 5 points in Ministry of Science and Higher Education parametric evaluation. § 8. 2) and § 12. 1. 2) 22.02.2019.

OThe Authors 2020;
This article is published with open access at Licensee Open Journal Systems of Nicolaus Copernicus University in Torun, Poland

Open Access. This article is distributed under the terms of the Creative Commons Attribution Noncommercial License which permits any noncommercial use, distribution, and reproduction in any medium, provided the original author (s) and source are credited. This is an open access article licensed under the terms of the Creative Commons Attribution Non commercial license Share alike.
(http://creativecommons.org/licenses/by-nc-sa/4.0/) which permits unrestricted, non commercial use, distribution and reproduction in any medium, provided the work is properly cited. The authors declare that there is no conflict of interests regarding the publication of this paper.

Received: 20.08.2020. Revised: 25.08.2020. Accepted: 12.09 .2020

\title{
Intravascular lithotripsy in the treatment of peripheral artery disease
}

\section{Michał Dobrzyński ${ }^{1}$, Jakub Czarnota ${ }^{1}$, Tomasz Skubel $^{1}$, Dorota Sokół ${ }^{1}$, Iga Dudek ${ }^{1}$, Malgorzata Drozd ${ }^{1}$, Halina Piecewicz-Szczęsna ${ }^{2}$}

${ }^{1}$ Student Research Circle at the Department of Epidemiology and Clinical Research Methodology, Medical University of Lublin

${ }^{2}$ Chair and Department of Epidemiology and Clinical Research Methodology, Medical University of Lublin

Michał Dobrzyński: https://orcid.org/0000-0002-1416-6568; mdobrzyski4@ gmail.com

Jakub Czarnota: https://orcid.org/0000-0003-2783-0349; kubbaa232@interia.pl Tomasz Skubel: https://orcid.org/0000-0001-7572-401X; tomasz.wojciech.skubel@ gmail.com

Małgorzata Drozd: https://orcid.org/0000-0002-0710-2451; drozd.malg@gmail.com Dorota Sokół: https://orcid.org/0000-0003-2059-7951; dorota.soko19606@gmail.com

Iga Dudek: https://orcid.org/0000-0002-8101-074X; iga.dudek6@ gmail.com Halina Piecewicz-Szczęsna: https://orcid.org/0000-0002-0573-7226; halpiec@wp.pl 


\begin{abstract}
Peripheral artery disease (PAD) is an atherosclerotic process leading to narrowing of the major distal arteries in relation to the aortic arch. The progressive closure of the artery leads to its narrowing, reduced blood flow and the appearance of intermittent claudication - the most common symptom among patients. Standard methods of management include drug treatment and lifestyle modification. In severe cases, surgical treatment is used, including intravascular lithotripsy (IVL). The aim of the study is to analyze the available data on the treatment of peripheral arterial disease with IVL. The work uses the latest data published on the PubMed platform. The efficacy of lithotripsy in the treatment of PAD has been confirmed in numerous publications. The therapeutic effect was obtained both using the lithotripsy procedure alone as well as in combination with other methods modifying atherosclerotic plaque. IVL is a safe method, the number of observed complications was negligible. After the patient is properly qualified for the procedure, IVL may become the therapy of choice in the treatment of PAD.
\end{abstract}

Key words: Intravascular lithotripsy, peripheral artery disease, cardiovascular diseases

\title{
Introduction
}

Peripheral artery disease (PAD) is a major cardiovascular disease that affected 202 million people worldwide in 2010. PAD is characterized by pathological distal vasoconstriction and is a serious cardiovascular disease with high mortality [1]. Factors increasing the risk of PAD include obesity (BMI> 30), smoking, hypercholesterolaemia, diabetes, hypertension and renal failure [2]. Peripheral arterial disease manifests itself by various symptoms depending on its localization. The most common include: intermittent claudication, atypical lower limb pain, acute limb ischemia, and critical limb ischemia caused by arterial lumen occlusion $[1,3]$. Peripheral artery disease is the third most common clinical manifestation of atherosclerosis after coronary artery disease and stroke [4]. Peripheral artery disease is the third most common clinical manifestation of atherosclerosis after coronary artery disease and stroke [4]. Ankle-brachial index (ABI) - the ratio of blood pressure at the ankle level to the highest blood pressure in the brachial artery - is a cheap and effective method used to diagnose PAD in primary care settings. It is very sensitive (90\%) and specific (98\%) [5]. Values below 0.9 suggest a diagnosis of peripheral arterial disease. Along with the longer duration of the disease, the risk of complications such as severe limb ischemia requiring amputation and heart attack increases [6]. Treatment of PAD includes both pharmacological therapy and interventions such as intravascular lithotripsy (IVL) [7, 8].

Originally, lithotripsy was mainly used in urology to treat kidney stones. The latest research proves the high effectiveness of intravascular lithotripsy to crush calcified atherosclerotic plaques. The undeniable advantage of intravascular lithotripsy is the crushing of both superficial and deep calcium. Also, during this procedure, calcium is not released into the bloodstream. Such action prevents embolism in distal vessels of smaller diameter [9]. 


\section{Aim of the study}

The aim of the study is to analyze the available data on the treatment of peripheral arterial disease with intravascular lithotripsy

\section{Materials and methods}

The materials used for the analysis come from the PubMed, UpToDate and SpringerLink platforms, which collect the latest medical articles.

\section{Intravascular lithotripsy procedure}

The endovascular lithotripsy procedure consists of 10 pulses ( 1 cycle) or 10 seconds (1 pulse per second). Depending on the degree of calcification of the lesion, a different number of lithotripsy cycles is used, but not more than 80 pulses (eight cycles). The selection of the diameter of the vascular catheter depends on the vessel diameter - the aim is to obtain a 1:1 ratio of the catheter-vessel diameter. Initially, the balloon is positioned in the vessel by inflating it to 4 atmospheres. The ultrasonic emitters are then activated using the trigger button. After delivering 10 pulses (one cycle), the balloon can be made up to 6 atmospheres and the symmetry of the vasodilatation can be assessed simultaneously by assessing the modification of calcification of the atherosclerotic lesion. Then the air from the balloon is carefully released in order to get rid of the air bubbles formed during the treatment. In the treatment of 1 lesion, it is recommended to use at least 2 IVL cycles. When the diameter of the vessel is narrower than the diameter of the lithotripsy balloon, the described procedure is combined with other methods of modifying the atherosclerotic plaque, eg rotablation or an NC balloon $[10,11]$.

\section{Review of the research considering intravascular lithotripsy}

In recent years, IVL has been used in the treatment of calcified atherosclerotic lesions in both large vessels such as the iliac arteries and in small coronary vessels.

\section{Intravascular lithotripsy in the iliac vessels}

In the study conducted by Armstrong et al. 2020, endovascular lithotripsy was successfully used in the treatment of calcification of the iliac arteries. 118 patients underwent treatment, including 101 with critical stenosis of the iliac arteries and symptoms of intermittent claudication. Severe calcifications were observed in $82 \%$ of patients. The mean artery stenosis was $83.1 \% \pm 13.4 \%$ with an average lesion length of $58.3 \mathrm{~mm} \pm 57.6 \mathrm{~mm}$. The catheter insertion procedure was successful in all patients. After lithotripsy, a vascular stent was additionally introduced in $72.9 \%$ of cases to supplement the therapy. The end result of the treatment was a reduction of stenosis to $12 \% \pm 12.1 \%$ of the vessel diameter. There were no adverse events related to the intervention [8].

In a study by Ristalli et al. 2019, vascular lithotripsy was used before the transcatheter aortic valve replacement (TAVR) and percutaneous mechanical circulatory support (MCS) procedures to decongest the iliac arteries for the insertion of a vascular catheter. The research group consists of 42 patients who underwent TAVR and 12 patients who underwent MCS. In both cases, full clinical success was observed, which meant successful TAVR and MCS 
preceded by lithotripsy. There were no life-threatening complications in any of the groups. IVL as a potential, safe method of restoring calcified femoral vessels prior to TAVR is confirmed in numerous case reports and a multicentre study, while data on the use of lithotripsy before MCS are limited and require further extensive research [12].

A case report of a 69-year-old patient with an aneurysm of the descending aorta who underwent TEVAR (Thoracic endovascular aortic repair) preceded by IVL was presented by Rosseel et.al in 2019. The limitation of this surgical method is the necessity to use large diameter instruments, which requires wide vascular access. The role of lithotripsy was to widen the lumen of the iliac artery to allow insertion of the tools needed for the TEVAR procedure. The procedure was performed without complications, and in control angiography, the correct vascular flow was obtained without signs of perforation or dissection [13]

\section{Intravascular lithrotripsy in the popliteal and superficial thigh arteries}

The DISRUPT PAD II study investigated the possibility of using IVL to reduce stenosis in the popliteal and superficial thigh arteries. 60 patients underwent treatment. $73.3 \%$ of the lesions concerned the superficial thigh artery, the remaining $26.7 \%$ of the stenoses were located in the popliteal artery. Mean arterial stenosis was $78.2 \% \pm 13.5 \%$. Procedural success was achieved in all patients undergoing the procedure. In some cases, additional interventions were applied: predilation (13.3\%), postdilation (3.3\%), anticoagulant filter $(3.3 \%)$ and stenting $(1.7 \%)$. The final postoperative arterial stenosis was $24.2 \%$. The vessel widened by an average of $3 \mathrm{~mm}$. Postoperative complications in the form of vascular wall dissection were observed in 9 cases, including 1 severe dissection (type D-F) [14].

In 2018, Brodmann et.al conducted a study on a group of 20 patients undergoing IVL surgery due to below the knee arterial stenoses. The primary safety endpoint was a composite of major adverse events through 30 days defined as death, myocardial infarction, need for emergency surgical revascularization of the target limb or amputation of the limb. Full therapeutic success was achieved in 19 cases. The composite of major adverse events at 30 days was $0 \%$ All patients achieved residual diameter stenosis $<50 \%$. In one case, type B dissection was observed, and in 2 cases it was necessary to insert a stent [15].

\section{Other treatments for PAD}

Treatment of PAD can be divided into: pharmacological and non-pharmacological.

In pharmacological management, multi-drug therapies are used depending on individual risk factors for peripheral arterial disease. The most commonly used are antiplatelet drugs (acetylsalicylic acid, clopidogrel), antihypertensive drugs (ACEI - angiotensin converting enzyme inhibitors, ARB - angiotensin receptor inhibitors), statins and hypoglycemic drugs [7].

Smoking cessation is recommended. Secondhand smoke exposure is an independent risk factor for PAD. High levels of secondhand smoke exposure were significantly associated with PAD [16]. Among initially healthy women, smoking is a potent risk factor for symptomatic PAD, an effect partially explained by subclinical inflammation. Smoking cessation substantially reduces PAD risk, but an increased occurrence of PAD persists even among former smokers who maintain abstinence [17]. 
Supervised exercises improve quality of life in patients with walking impairment. A supervised exercise therapy (SET) should be considered as a treatment option for claudication before surgical or endovascular revascularization [7].

Lithotripsy shows an advantage in highly calcified lesions, which are a great challenge for other surgical methods. Apart from lithotripsy, surgical treatment of PAD includes: angioplasty with stent implantation, rotablation or an excimer laser [18].

\section{Restrictions on the use of IVL}

Some lesions have features that prevent an effective intravascular lithotripsy procedure:

- winding course of the vessel,

- critical narrowing of the vessel's lumen,

- condition after multiple stenting of the lesion,

- unfavorable shape of atherosclerotic plaque.

These clinical situations do not allow for the correct insertion and positioning of the lithotripsy balloon [19].

In order to reduce the risk of side effects associated with the IVL procedure and to correctly qualify the patient for the procedure, it is worth making early imaging of the vessels being the target of the therapy. The optimal choice is to use optical coherence tomography. It also enables the planning of possible adjuvant therapy which means the use of other plaque modifying methods, which will increase the effectiveness of the procedure [10].

\section{Conclusions}

In the light of the presented studies, intravascular lithotripsy is a promising method of treatment of calcified atherosclerotic lesions. It is associated with a high rate of therapeutic effectiveness and a low risk of complications. It is a universal method, which can be used with equally high efficiency in vessels of various sizes, both coronary and peripheral. There is a need to compare lithotripsy with other available plaque modifying methods to determine the place of IVL in modern medicine.

\section{References}

[1] P. Song et al., "Global, regional, and national prevalence and risk factors for peripheral artery disease in 2015: an updated systematic review and analysis," Lancet Glob. Heal., vol. 7, no. 8, pp. e1020-e1030, Aug. 2019, doi: 10.1016/S2214-109X(19)30255-4.

[2] L. Norgren, W. R. Hiatt, J. A. Dormandy, M. R. Nehler, K. A. Harris, and F. G. R. Fowkes, "InterSociety Consensus for the Management of Peripheral Arterial Disease (TASC II)," Journal of Vascular Surgery, vol. 45, no. 1 SUPPL. J Vasc Surg, Jan-2007, doi: 10.1016/j.jvs.2006.12.037.

[3] F. G. R. Fowkes, V. Aboyans, F. J. I. Fowkes, M. M. McDermott, U. K. A. Sampson, and M. H. Criqui, "Peripheral artery disease: Epidemiology and global perspectives," Nature Reviews Cardiology, vol. 14, no. 3. Nature Publishing Group, pp. 156-170, 01-Mar-2017, doi: 10.1038/nrcardio.2016.179.

[4] F. G. R. Fowkes et al., "Comparison of global estimates of prevalence and risk factors for peripheral artery disease in 2000 and 2010: A systematic review and analysis," Lancet, vol. 382, no. 9901, pp. 1329-1340, Oct. 2013, doi: 10.1016/S0140-6736(13)61249-0.

[5] T. W. Rooke et al., "2011 ACCF/AHA focused update of the guideline for the management of patients with peripheral artery disease (Updating the 2005 Guideline): A report of the american college of cardiology foundation/American Heart Association task force on practice guidelin," Circulation, vol. 124, no. 18, pp. 20202045, Nov. 2011, doi: 10.1161/CIR.0b013e31822e80c3. 
[6] K. Ouriel, "Peripheral arterial disease," in Lancet, 2001, vol. 358, no. 9289, pp. 1257-1264, doi: 10.1016/S0140-6736(01)06351-6.

[7] R. Morcos et al., "The Evolving Treatment of Peripheral Arterial Disease through Guideline-Directed Recommendations,” J. Clin. Med., vol. 7, no. 1, p. 9, Jan. 2018, doi: 10.3390/jcm7010009.

[8] E. J. Armstrong et al., "Intravascular lithotripsy for treatment of calcified, stenotic iliac arteries: A cohort analysis from the Disrupt PAD III Study," Cardiovasc. Revascularization Med., Mar. 2020, doi: 10.1016/j.carrev.2020.02.026.

[9] C. S. Dini et al., "Intravascular lithotripsy for calcific coronary and peripheral artery stenoses," EuroIntervention, vol. 15, no. 8, pp. 714-721, Oct. 2019, doi: 10.4244/eij-d-18-01056.

[10] M. N. T. Forero and J. Daemen, "The coronary intravascular lithotripsy system," Interv. Cardiol. Rev., vol. 14, no. 3, pp. 174-181, Dec. 2019, doi: 10.15420/icr.2019.18.R1.

[11] G. Kassimis et al., "How Should We Treat Heavily Calcified Coronary Artery Disease in Contemporary Practice? From Atherectomy to Intravascular Lithotripsy," Cardiovascular Revascularization Medicine, vol. 20, no. 12. Elsevier Inc., pp. 1172-1183, 01-Dec-2019, doi: 10.1016/j.carrev.2019.01.010.

[12] F. Ristalli et al., "Role of Lithotripsy for Small Calcified Iliacs in the Era of Big Devices," Current Cardiology Reports, vol. 21, no. 11. Springer, pp. 1-8, 01-Nov-2019, doi: 10.1007/s11886-019-1245-2.

[13] L. Rosseel, O. De Backer, L. Søndergaard, and G. Bieliauskas, "Intravascular iliac artery lithotripsy to enable transfemoral thoracic endovascular aortic repair," Catheter. Cardiovasc. Interv., vol. 95, no. 3, pp. E96E99, Feb. 2020, doi: 10.1002/ccd.28379.

[14] M. Brodmann et al., "Primary outcomes and mechanism of action of intravascular lithotripsy in calcified, femoropopliteal lesions: Results of Disrupt PAD II," Catheter. Cardiovasc. Interv., vol. 93, no. 2, pp. 335-342, Feb. 2019, doi: 10.1002/ccd.27943.

[15] M. Brodmann, A. Holden, and T. Zeller, "Safety and Feasibility of Intravascular Lithotripsy for Treatment of Below-the-Knee Arterial Stenoses," J. Endovasc. Ther., vol. 25, no. 4, pp. 499-503, Aug. 2018, doi: $10.1177 / 1526602818783989$.

[16] L. Lu, D. F. Mackay, and J. P. Pell, "Association between level of exposure to secondhand smoke and peripheral arterial disease: Cross-sectional study of 5686 never smokers," Atherosclerosis, vol. 229, no. 2, pp. 273-276, Aug. 2013, doi: 10.1016/j.atherosclerosis.2013.05.015.

[17] D. Conen et al., "Smoking, Smoking Cessation and Risk of Symptomatic Peripheral Artery Disease in Women: A Prospective Study,” Ann. Intern. Med., vol. 154, no. 11, p. 719, 2011, doi: 10.1059/0003-4819-15411-201106070-00003.

[18] K. Nakabayashi et al., "Combined Use of Excimer Laser and High-Speed Rotational Atherectomy to Overcome a Severely Calcified Lesion in Endovascular Therapy," Case Rep. Vasc. Med., vol. 2019, 2019, doi: $10.1155 / 2019 / 1719035$.

[19] G. Chen, B. Zrenner, and S. A. Pyxaras, "Combined Rotational Atherectomy and Intravascular Lithotripsy for the Treatment of Severely Calcified in-Stent Neoatherosclerosis: A Mini-Review," Cardiovasc. Revascularization Med., vol. 20, no. 9, pp. 819-821, Sep. 2019, doi: 10.1016/j.carrev.2018.10.007. 